\title{
Genealogía, biopolítica y tanatopolítica : La cuestión de la vida en la Filosofía Biopolítica*
}

Genealogy, biopolitics and thanatopolitics: the question of life in Biopolitical Philosophy

\author{
Por: González, Daiana Marlén* \\ Universidad Nacional del Nordeste \\ E-mail: marlengonzalez9679@gmail.com
}

Fecha de recepción: 15/03/2020

Fecha de aprobación: 30/04/2020

DOI: 10.30972/ach.054316

\section{Resumen:}

Este trabajo reúne una serie de aportes al debate biopolítico de acuerdo con una formulación expuesta en la obra Bios de Roberto Espósito. El desarrollo del texto se centra en la relación entre la noción de biopolítica y tanatopolítica. Espósito realiza una lectura de Michel Foucault, en la que expone como principal eje de debate la problemática relación de una biopolítica que deviene incesantemente tanatopolítica. Aquí se proponen una serie de contribuciones que la obra de Nietzsche puede arrojar sobre este debate. Se trata especialmente de aportes genealógicos de Nietzsche, presentes en la obra Genealogía de la Moral, entre otras del filósofo alemán. Como así también se centra la atención en el concepto de vida, ideal ascético y la ética nietzscheana.

Palabras claves: Genealogía; Nietzsche; Espósito; Vida; Biopolítica

\footnotetext{
* El presente trabajo surgen en marco de la cátedra Seminario II. Análisis de un problema filosófico del año 2019.

*Estudiantx del Profesorado y Licenciatura en Filosofía de la Facultad de Humanidades (UNNE).
} 


\section{Abstract.}

This work brings together a series of contributions to the biopolitical debate in accordance with the proposal presented in the work Bios by Roberto Espósito. The development of the text focuses on the relationship between the notion of biopolitics and thanatopolitics. Espósito reads Michel Foucault and argues that the main discussion is based on the problematic relationship between a biopolitics that ceaselessly turns into thanatopolitics. Here we proposed a series of contributions that Nietzsche's work can make to this debate. These are especially the genealogical contributions of Nietzsche, present in his work On the Genealogy of Morality, among others by the German philosopher. As well, attention is also focused on the concept of life, ascetic ideal and Nietzscheana ethics.

Keywords: Genealogy; Nietzsche; Espósito; Life; Biopolitics

\section{Cómo citar este artículo:}

APA: González, D. M. (2020). Genealogía, biopolítica y tanatopolítica : La cuestión de la vida en la Filosofía Biopolítica, Acheronta, 5, 27-56. Recuperado de: (agregar dirección web)

\section{Introducción}

El presente trabajo se da en el marco de la cursada de Seminario II: Análisis de un problema filosófico. El problema elegido en este caso es la relación entre biopolítica y tanatopolítica a partir del planteo de Roberto Espósito y pretende recoger los aportes que la obra de de Friedrich Nietzsche pueden hacer en este campo en general y al problema en particular. La obra de Nietzsche es retomada por varios/as autores/as vinculados a los estudios biopolíticos, entre ellos destacamos al mismo Espósito, Vanessa Lemm y Mónica Cragnolini, entre otros. Si bien en este caso nos servimos de algunas líneas de pensamientos de estos autores, el principal insumo teórico nos está 
dado en los aportes de la obra de de Nietzsche. Para el tratamiento de este tema en particular nos centramos en un recorrido amplio que incluye varias obras, de las cuales destacamos sólo los aspectos que se consideraron oportunos para el abordaje de esta problemática. Se recorren temas vinculados a la crítica cultural a la modernidad, el ideal ascético, la noción de vida y los aportes teórico-metodológicos de la Genealogía.

La importancia de una investigación de este tema radica en la actualidad del campo de estudio de la biopolítica, que suscita variados estudios y debates contemporáneos. De este modo, el uso del término biopolítica responde a necesidades propias del contexto social y político como del desarrollo epistemológico de las ciencias y disciplinas; a esto hace referencia Cragnolini cuando menciona (2009): “(...)cómo las problemáticas contemporáneas no pueden ser abordadas si no es desde diferentes disciplinas que entran en cruces y que aportan sus campos semánticos y metodologías." (p.186). Con respecto al marco teórico específico y por tratarse de un trabajo filosófico, los insumos para la investigación fueron en su totalidad bibliográficos y la metodología reunirá análisis críticos conceptuales y hermenéuticos de los autores trabajados.

Para hacer más clara la introducción, mencionaremos brevemente los objetivos que nos planteamos en torno a la misma. El trabajo tiene dos objetivos amplios y generales vinculados a "Identificar y analizar los elementos que constituyen el ideal ascético en la obra de Nietzsche y su dinámica de acuerdo con el concepto de vidamuerte, autosuperación-conservación, y además la necesidad de "Plantear y desarrollar los aportes que este concepto puede brindar al problema de la relación entre biopolítica y tanatopolítica en Espósito." En cuanto a objetivos más específicos, se desprenden de los anteriores tanto la pregunta sobre la " la existencia de elementos propios de una biopolítica afirmativa y negativa en el ideal ascético nietzscheano". Así como también la posibilidad de "Enunciar la necesidad de una interpretación no binaria y excluyente del concepto nietzscheano de vida de acuerdo a la identificación de conceptos tanatológicos de la misma." 
Abril 2020.

ISSN 2344-9934

A fin de lograr los objetivos propuestos, nos hemos visto en la necesidad de introducir breves cambios en la estructura del trabajo en lo que respecta al orden de los capítulos con sus respectivos temas a tratar. Este cambio se debe en parte a una necesidad de estructurar lógicamente los contenidos para la comprensión del lector. Por otro lado, a que en el proceso mismo de escritura una exposición en el orden que consignamos finalmente resultaba más beneficiosa para el último capítulo que resulta una síntesis y recoge los elementos ya expuestos en los tres capítulos anteriores. Así, el orden cronológico y lógico que nos resultó más propicio para la comunicación de estos resultados de investigación se pueden esbozar de la siguiente manera. El primer capítulo titulado El planteo de Esposito: ¿Qué es la biopolítica? La relación entre biopolítica y tanatopolítica expone de forma breve y clara los planteos en torno a la relación entre biopolítica y tanatopolítica que el autor ofrece en Bios. Esto a partir de la lectura que el autor italiano realiza de Michel Foucault. El segundo capítulo de este escrito constituye una lectura sistemática de La genealogía de la Moral y un análisis de Vergara Henríquez sobre la misma, asimismo señala los aportes que esta obra hace al campo de la biopolítica y pone un énfasis especial en el concepto de ideal ascético como ideal de décadence por excelencia. El tercer capítulo, retoma la cuestión clave si de lo que se trata es pensar la relación entre poder y vida, la noción misma de vida para Nietzsche, a partir de la lectura combinada de varias obras del autor y de un artículo de García Granero que problematiza esta noción. El cuarto y último capítulo pretende ser una síntesis y lectura crítica-reflexiva sobre los capítulos anteriores. Por lo que se retoma el planteo de Espósito con los aportes y reflexiones que surgieron a partir de la lectura nietzscheana para dicho fin.

\section{El planteo de Esposito: ¿Qué es la biopolítica? La relación entre biopolítica y}

\section{tanatopolítica}

En Bios. Biopolítica y filosofía, el filósofo Roberto Espósito presenta, mediante una exposiciones secuencial de casos, el problema siempre en tensión de lo que él 
mismo denomina una "manifiesta superposición entre declarada defensa de la vida y efectiva producción de muerte." (Esposito, 2006, p.10). Se declara aquí entonces, el problema de la estrechez siempre en tensión y en cuestión entre vida y muerte, o más precisamente entre biopolítica y tanatopolítica. Dos términos, estos últimos que al menos en su nivel teórico parecieran presentar dos caras diferentes de expresión del poder sobre la vida. Este problema, abordado desde una perspectiva filosófica, es puesto en el centro del debate y es aquí donde Espósito se sirve de las respuestas dadas por Michel Foucault. Sin embargo, no duda en resaltar el carácter poco definitivo de las respuestas del francés, lo que da lugar a intento de un análisis más exhaustivo por parte de Espósito.

Si bien la introducción que hace el autor a su obra, tiene por finalidad expresar de manera mucho más clara y concentra la tensión entre un poder de la vida y un poder sobre la vida; e incluso más allá del intento de ilustrar la problemática mediante la exposición de los casos particulares e históricos allí expuestos, estos no serán retomados aquí. La exposición se dirige más bien, al nivel conceptual que se genera a partir de estos acontecimientos. Como menciona el autor, en el centro de todos ellos:

“(...) está la noción de biopolítica. Sólo basándose en ella es posible encontrar, para acontecimientos como los expuestos, que escapan a una interpretación más tradicional, un sentido global que vaya más allá de su mera manifestación. En verdad, ellos devuelven una imagen extrema, pero ciertamente no inexacta, de una dinámica que a esta altura involucra a todos los grandes acontecimientos políticos de nuestro tiempo." (Espósito,2006, p. 15)

A partir de este planteo, el centro de atención pone su foco en la obra de Michel Foucault, con especial atención a los escritos de la década del '70. El autor lo hace a partir de una pregunta que sirvió de base para este trabajo y que lo recorre completamente ¿por qué, al menos hasta hoy, una política de vida, amenaza siempre con volverse acción de muerte? (Esposito, 2006, p. 16). Ante esto, ya se vislumbra una 
Abril 2020.

ISSN 2344-9934

primera advertencia de Espósito: Michel Foucault nunca dio una respuesta definitiva a este problema.

De esta problemática, en la que él autor francés nunca arriesgó una respuesta definitiva, se desprenden dos concepciones que se plantan como contrapuestas, la de una biopolítica afirmativa y hasta eufórica en términos de Espósito, y una rotundamente negativa. Sobre estas dos concepciones se desplaza el problema aquí expuesto y tiene en cuenta aristas que van desde el plano semántico, lógico, político e histórico. Como menciona el pensador italiano, este planteo va más allá de un análisis meramente filológico de los términos expuestos: biopolítica, poder, vida. Aunque no por ello, es menos necesaria una aclaración de dichos términos.

Uno de los puntos más importantes y cuyo eje analítico será tenido en cuenta de manera especial, se trata de la relación entre biopolítica y modernidad. En este punto, las preguntas frecuentes se cuestionan si “¿La biopolítica precede a la modernidad, la sigue, o coincide temporalmente con ella? ¿Tiene una dimensión histórica, epocal, u originaria?" (Espósito, 2006, p.17). Ante este problema, Foucault no da una respuesta acabada, Esposito se atreve a responder a partir de lo que llama el paradigma inmunitario que a partir de una proyección negativa de la vida, la biopolítica puede ser vinculada en su surgimiento con la modernidad (Espósito, 2006, p. 17). Si bien se opta por esta vinculación entre biopolítica y modernidad, el autor no tarda en aclarar que ésta no responde a una exclusividad de rasgos biopolíticos presentes en la modernidad, ya que también es posible rastrear aspectos propios de la biopolítica en otras épocas históricas. Y esta aclaración no es menor, teniendo en cuenta que permite avanzar un poco más sobre un posible punto de unión entre biopolítica y modernidad, el arriesga una respuesta, según él “(...)sólo la modernidad hace de la autoconservación del individuo el presupuesto de las restantes categorías políticas, desde la de soberanía hasta la de libertad." (Espósito, 2006, p.17-18).

Pero volvamos al análisis sobre Foucault. Espósito hace una exposición muy clara acerca de autores que ya tuvieron presente en sus obras este problema biopolítico. En todos ellos se manifiesta la polisemia con que el término biopolítica es 
usado, lo que da cuenta de la dificultad para encontrar una referencia clara del mismo, así como también el riesgo de la pérdida de identidad de lo que se significa con biopolítica. Teniendo en cuenta esto, el autor francés no es considerado el único en plantear estas cuestiones, ni el primero, es más, para Espósito Foucault culmina un recorrido ya iniciado por una importante cantidad de autores modernos. Ahora bien ¿qué es lo que da al planteo foucaultiano su particularidad y originalidad? Aquí ya se vislumbran las influencias de Nietzsche, en este caso dado por la cuestión metodológica, pues “(...) detrás de su específica perspectiva biopolítica, y dentro de ella, esté en primer lugar la genealogía nietzscheana." (Epósito, 2006, p.41). Esta primera referencia a Nietzsche está relacionada con el tratamiento que hace Foucault de los conceptos o categorías modernas en torno al poder y la vida, en especial la singularidad con la que plantea el problema entre poder soberano y biopolítica y la reestructuración de los planteos realizados hasta entonces. De ello se desprende toda una preocupación teórica que obtiene su mayor problematización en los años '70, y que con sus bifurcaciones y continuidades tiene como centro de análisis la cuestión de la vida. Espósito (2006), señala acertadamente que:

La vida y nada más que la vida -las líneas de desarrollo en las que se inscribe o los vórtices en los que se contrae - es tocada, atravesada, modificada aun en sus fibras íntimas por la historia. Esta era la lección que Foucault había extraído de la genealogía nietzscheana, dentro de un marco teórico que reemplazaba la búsqueda del origen, o la prefiguración del fin, por un campo de fuerzas desencadenado por la sucesión de los acontecimientos y por el enfrentamiento de los cuerpos. (p. 49)

Además, una alusión a Darwin, del que más adelante nos ocuparemos brevemente, pero allí en relación a Nietzsche. La influencia darwiniana dirá Espósito (2006) está en "(...) haber reconocido también en la vida los signos, brechas y azares de la historia". (p. 49). Esto supone además el entrecruzamiento constante entre vida e historia, al que hace referencia la bio-historia de Foucault, no es más ni menos, que la vida 
Abril 2020.

pudiendo ser afectada por la historia, los poderes, saberes, acontecimientos, pero además ella misma adentrándose enteramente en dicha historia. Queda como resultado, en primer lugar, la cuestión tal vez más determinante del planteo foucaultiano, la vida entendida no como algo natural e inalterable, no como supuesto de posteriores categorías políticas, sino como algo puesto, en palabras de Espósito. Esto a su vez, y como se podrá vislumbrar, dificulta cualquier interpretación monocausal sobre el asunto y, aquí también se observan influencias nietzscheanas sobre las cuales ahondaremos más adelante. La primera tarea entonces, aunque no haya llegado a una respuesta definitiva, queda ya marcada por Foucault, la no reducción del estudio al ámbito exclusivo de la vida como un en sí, ni de la historia como algo externo a sus saltos, desarrollos y pulsiones.

En este punto y retomando lo dicho, se abre la pregunta acerca del cómo es que la vida afecta a la historia y la historia a la vida, cuál es el efecto y cómo es la dinámica de la biopolítica. No sorprende en esta instancia que el discurso foucaultiano arriesgue diferentes respuestas a estas formulaciones, no al menos, teniendo en cuenta la perspectiva metodológica en la que se inscribe y las influencias teóricas que lo atraviesan. La cuestión de la vida en el centro de atención, o del bíos en el lenguaje de Esposito oscila al menos, entre dos respuestas distintas. Espósito lee en Foucault, la tensión entre una posición que sitúa a la biopolítica con la producción de la subjetividad o la producción de muerte. Aquí lo expresa de forma clara:

Ambas - con respecto a la vida - constituyen más que dos posibilidades. Son a un tiempo su forma y su fondo, su origen y su destino, pero conforme a una divergencia que parece no admitir mediaciones: una u otra. O la biopolítica produce subjetividad, o produce muerte, $\mathrm{O}$ torna sujeto a su propio objeto, o lo objetiviza definitivamente. $\mathrm{O}$ es política de la vida, o sobre la vida. (Espósito, 2006, p. 53)

Una de las problemáticas que atraviesa el planteo foucaultiano hace referencia a la relación entre el paso de un poder soberano a la biopolítica. El pensador francés 
parece ver en los modos de sujeción propios del poder disciplinario una marcada diferencia para con los mecanismos del poder soberano. Tiene incluso para la aparición de esta nueva forma del ejercicio del poder una demarcación histórica que va desde los siglos XVII y XVIII. Si se puede definir a nivel conceptual como Foucault entiende esta diferencia, se resume en la célebre frase "Podría decirse que el viejo derecho de hacer morir o dejar vivir fue reemplazado por el poder de hacer vivir o de rechazar hacia la muerte." (Foucault, 2007, p. 167).

Como acertadamente marcará Esposito, la línea divisoria entre ambos poderes resulta de el lugar o la posición estratégica que ocupa la vida en cada caso. Si en el poder soberano la fuerza y el poder del soberano se mostraba especialmente en la posibilidad de producir la muerte, la vida de los súbditos aparece como un resto o un residuo que es posible negar si el soberano así lo entiende. En cambio, en el contexto de la biopolítica la muerte aparece como el límite de una vida que debe conservarse y desarrollarse. Más allá de todo el desarrollo foucaultiano sobre la biopolítica y los poderes de disciplinamiento y control lo que se intenta aquí es mostrar esta primer respuesta, que como se puede observar, este opone punto por puntos las dos formas de poderes.

Una segunda respuesta a este problema se ensaya a partir de la idea de una continuidad casi germinal entre el poder soberano y la biopolítica. Existe la sensación en este caso de la presencia de una mirada más genealogista que la anterior. En primer lugar, esta idea de continuidad sugiere que el poder soberano es el fondo de donde emerge la biopolítica, el punto está en establecer si existe o no una superación total del mismo. Otro de los aspectos que nos interesa señalar es que, en la idea de una posible oposición entre ambos regímenes se observa una formulación conceptual que desde el punto de vista del lenguaje parece ser posible. Es decir, la idea de una identidad del poder soberano que se distingue completamente de la biopolítica. El problema radica como hemos visto, en que los hechos en su desencadenamiento, ponen en cuestión dicha diferencia y discontinuidad. Lo que nos interesa plantear aquí 
Abril 2020.

es que si bien el surgimiento de una nueva forma de control de los cuerpos individuales y luego de la población sufrieron cambios, esto no se traduce necesariamente en una forma de biopolítica afirmativa y mucho menos la desestimación total de la tanatopolítica.

Esta última cuestión, es una paradoja que como señalamos Foucault no terminó de resolver. Esposito nos devuelve el problema bajo el planteo ya descrito. Ante toda esta formulación, nos serviremos de ahora en más de Nietzsche, autor que Espósito abraza y lo ubica entre la primera y segunda inmunización de su planteo biopolítico. No será este nuestro caso, se intentará realizar un breve recorrido de varios puntos claves de la obra nietzscheana que nos permitan identificar y exponer los aportes que esta puede tener a la problemática relación entre biopolítica y tanatopolítica.

Uno de los últimos puntos claves que queremos mencionar, es la dificultad misma que se presenta en torno a la noción de biopolítica. Ante ella Espósito intenta hacer notar incansablemente la necesidad lógico semántica de esclarecer los términos en los que se habla de biopolítica. Dirá por ejemplo que:

Biopolítica no remite sólo, o predominantemente, al modo en que, desde siempre, la política es tomada -limitada, comprimida, determinada- por la vida, sino también, y sobre todo, al modo en que la vida es aferrada, desafiada, penetrada por la política. (Espósito, 2006, p. 51)

Asimismo resaltará en otros momentos la necesidad de pensar los términos vida y poder tanto en su dimensión particular y separada como un su relación estrecha y determinación o codeterminación entre ambos. Ante todas estas ambigüedades, que no resultan un obstáculo metodológica y conceptual fácil de superar, hemos decidido optar por señalar como punto final, la diferencia entre biopolítica y biopoder para una mayor claridad: 
¿Qué significa el gobierno político de la vida? ¿Debe entenderse que la vida gobierna la política, o bien que la política gobierna la vida? ¿Se trata de un gobierno de o sobre la vida? Esta misma disyuntiva conceptual puede expresarse mediante la bifurcación léxica entre los términos «biopolítica» y «biopoder», empleados indistintamente en otras circunstancias; por el primero se entiende una política en nombre de la vida, y por el segundo, una vida sometida al mando de la política. (Espósito, 2006, p. 26)

Cerramos por ahora con el planteo de Espósito y nos adentramos a los análisis de conceptos exclusivamente nietzscheanos, para luego volver al planteo y formular los aportes que dichas nociones pueden brindar a este problema.

\section{Un análisis genealógico: El ideal ascético en Nietzsche. Elementos, dinámica y} funcionamiento

Como se menciona en el título de nuestro trabajo, uno de los principales conceptos que retomamos aquí como aporte a la problemática analizada es el de ideal ascético. Este concepto es clave en la obra de Friedrich Nietzsche y es uno de los pilares sobre los que dicho autor ejerce una crítica radical de los valores morales y la cultura occidental.

Para llegar a una descripción clara y precisa de este concepto nos serviremos de una lectura secuencial y sistemática de la obra conocida como La Genealogía de la Moral y además del artículo de Vergara Henríquez: El "sacerdote asceta" y el sacrodominio del valor. Nietzsche y la genealogía de la moral, que brinda una lectura muy sistemática de la misma.

Para los lectores de Nietzsche, es un hecho conocido la importancia y la fuerza que tiene este escrito para el estudio de valores morales, así como también sus 
Abril 2020.

inmensos aportes metodológicos. Mencionamos brevemente algunas cuestiones a tener en cuenta. Este libro nos sirve de insumo para el trabajo no solo por su aporte en referencia al ideal ascético, al cual le dedica en su totalidad el tercer tratado del mismo, sino que además y a diferencia de otros escritos nietzscheanos, está dotado de una sistematicidad y un desarrollo temático favorable. Por otro lado, el ideal ascético es tratado en varios de los escritos del pensador alemán por lo cual los mismos también serán retomados cuando la exposición así lo requiera. Por último, en nuestro tratamiento del ideal ascético, que incluye una historización del mismo, es necesaria también, como se verá al final del capítulo una contextualización del mismo, pensándolo a partir de coordenadas más actuales ya que está todo el tiempo presente la importancia de unirlo a un aporte sobre la cuestión de la tanatopolítica y la biopolítica. Esto dará lugar en los siguientes capítulos a relacionarlo con una crítica cultural de la modernidad, el nihilismo, el trabajo, el Estado, etc.

Vergara Enriquez (2011) dirá qué "La fórmula precisa que expresa el diagnóstico nietzscheano sobre la cultura occidental es que está enferma moralmente, debido a estar escindida entre los elementos racionales del pensamiento y las raíces terrenales o sensibles de la vida misma en cuanto voluntad de poder." (pp. 129-130) Estamos en condiciones de afirmar por lo tanto, que uno de los mayores diagnósticos de la cultura occidental se establecen en la genealogía de la moral, si bien no Nietzsche no comienza aquí su crítica, este estudio estará especialmente dedicado según su autor a una indagación sobre la moral, para ser más precisos, Nietzsche (2005) se pregunta:

¿en qué condiciones se inventó el hombre esos juicios de valor que son las palabras bueno y malvado? y ¿qué valor tienen ellos mismos? ¿Han frenado o han estimulado hasta ahora el desarrollo humano? ¿Son un signo de indigencia, de empobrecimiento, de degeneración de la vida? ¿O, por el contrario, en ellos se manifiestan la plenitud, la fuerza, la voluntad de la vida, su valor, su confianza, su futuro? (p. 24) 
A estas preguntas, al problema filosófico sobre el valor de los valores morales Nietzsche responde con la genealogía, estudio que incluye tanto a la filosofía, la fisiología, la filología y los estudios históricos. Ante esto, es preciso realizar un par de precisiones terminológicas. ¿Qué entendemos por moral? ¿En qué consiste el método genealógico que la estudia? Podemos afirmar que en Nietzsche la palabra moral es polisémica. De ella Nietzsche (2005) dirá: "(la moral como consecuencia, como síntoma, como máscara, como tartufería, como enfermedad, como malentendido; pero también la moral como causa, como medicina, como estímulo, como freno, como veneno)" (p.28). Pero de acuerdo con el objetivo de la obra, cuando Nietzsche habla de moral y ejerce la crítica sobre la misma esta reviste un carácter negativo para la vida, en palabras de Cano (2015):

Por ésta (la moral), Nietzsche entiende no solo la estructura dualista de la metafísica (la separación entre mundo verdadero y mundo aparente), sino también su insuficiente -y por ello falsa- superación a través del discurso científico. Moral, en una palabra es toda problematización que, insatisfecha con la ambivalencia esencial de la vida, pretende infructuosamente escapar de ella al abrigo de un mundo ideal. Por medio de este desdoblamiento ficticio entre lo real y lo ideal, la imperfección de la existencia resulta sublimada hacia otro nivel del ser, con una grave consecuencia: la culpa de la vida. (p. 52)

Con respeto a la Genealogía como método, más allá de las muchas apropiaciones e interpretaciones que pueden realizarse sobre la misma, diremos brevemente que se trata de una "Metodología histórica orientada a la crítica de los valores morales, cuyo interés se cifra en el desciframiento de las construcciones ideales como síntomas corporales." (Cano, 2015, p.99).

Dicho esto, pasemos a considerar la crítica nietzscheana a la moral. El primer tratado de la Genealogía expone el surgimiento de los conceptos bueno-malo, bueno- 
Abril 2020.

malvado. Lo que el autor llama doble prehistoria de los sentimientos morales tiene que ver que con estas dos formas de valorar. En un primer momento de este proceso, los valores que imperaban tenían que ver con la separación bueno-malo, propia de una cultura fuerte y aristócrata. Lo bueno se vinculaba con un movimiento espontáneo de autopercepción por parte de los hombres aristocráticos. Estos se consideraban a sí mismos buenos, en un sentido estamental, que también estaba vinculado con rasgos de su carácter y templanza. En cambio, malo designaba a los plebeyos, vulgares, bajos. Lo que sucede luego, es una transvaloración de dichas denominaciones que dará lugar al binomio bueno-malvado.

La transvaloración de los valores consiste según Nietzsche en un proceso en el cual los valores morales como bueno y malo, que en su origen tuvieron significaciones completamente opuestas a las de su época actual, se transforman y adquieren otro valor y significado. En un primer momento el término bueno (gut) designaba la valoración y el aprecio que tenían de sí mismos y de sus acciones los hombres nobles, poderosos, aristócratas que se consideraban superiores y opuestos al concepto de malo (schlecht), que designaba lo simple como todo lo vulgar, bajo y plebeyo. Este sentimiento es lo que Nietzsche llama pathos de la distancia y en él tienen su génesis los valores morales de bueno y malo. Así lo bueno designaba los rasgos y el rango propios de los espíritus nobles estos eran los valerosos, superiores, veraces, elevado,de constitución física fuerte, etc., aquellos de carácter caballeresco-aristócrata que son amantes de las aventuras, la guerra y toda actividad en donde se exprese su libertad y fortaleza. En contraposición a ellos, designaban lo malo como todo lo típico del espíritu plebeyo, bajo y vulgar, que es el hombre débil, mentirosos, impotentes.

Este proceso se da especialmente a partir de las castas aristocráticassacerdotales, en donde la manera de valorar difiere mucho de la de los espíritus nobles, el autor señala que: "Desde el comienzo hay algo no sano en tales aristocracias sacerdotales y en los hábitos en ellas dominantes (...)" (Nietzsche, 2005, 44). Esta diferencia está marcada fisiológicamente: 
Los juicios de valor caballeresco-aristocráticos tienen como presupuesto una constitución física poderosa, una salud floreciente, rica, incluso desbordante, junto con lo que condiciona el mantenimiento de la misma, es decir, la guerra, las aventuras, la caza, la danza, las peleas y, en general, todo lo que la actividad fuerte, libre, regocijada lleva consigo. La manera noble-sacerdotal de valorar tiene -lo hemos visto- otros presupuestos: ¡las cosas les van muy mal cuando aparece la guerra! (Nietzsche, 2005, p.45)

Para Nietzsche el pueblo y los sacerdotes judíos (del cual el cristianismo va a ser su heredero) son los causantes de la rebelión de los esclavos, en esta se produce la caída de la aristocracia y la transvaloración de los valores de la misma; según el autor: "La rebelión de los esclavos en la moral comienza cuando el resentimiento mismo se vuelve creador y engendra valores." (Nietzsche, 2005, p.50). Estos son incapaces de una auténtica reacción, la que está vinculada al accionar a partir de sí; su reacción es la típica del espíritu del resentimiento, por la cual solo valoran negando el valor que se le opone, su hacer es una negación. (Nietzsche, 2005).

Como consecuencia de esto surge la caída de toda moral noble y superior, representa un retroceso en la humanidad. El sacerdote como inventor de la religión, principal representante del ideal ascético, el deseo de nada (de Dios), ha vuelto al hombre un animal interesante, un alma profunda, se crea el valor de lo malvado. A partir de ahora, estos creadores de valores decadentes valoraran todo lo empobrecido, menesteroso, débil, sufriente, enfermo como lo bueno en sí y todo lo fuerte, poderoso, sano, insaciable, cruel como lo malvado. La diferencia de esta clase de valoración con respecto al binomio bueno-malo radica en que en la primera opera a partir del concepto de voluntad libre. Según esta, los fuertes pueden elegir ser algo distinto a lo que son, de ahí el juicio moral y el calificativo de malvados a quien no adopte la moral decadente. De ello Nietzsche (2005), dirá que: “Exigir de la fortaleza que no sea un querer-dominar, un querer-sojuzgar, un querer-enseñorearse, una sed de enemigos y de resistencias y de triunfos, es tan absurdo como exigir de la debilidad que se exteriorice como fortaleza. " (p.59) 
La consecuencia de este proceso de adiestramiento está a la vista “(...) el que el hombre manso, el incurablemente mediocre y desagradable haya aprendido a sentirse a sí mismo como la meta y la cumbre, como el sentido de la historia, como el "hombre superior;" (Nietzsche, 2005, p.57) es lo que representa una fatalidad en la historia, a partir de esto el hombre tiene aversión por la humanidad. Por ello "Actualmente la visión del hombre cansa-¿qué es hoy el nihilismo si no es eso?...Estamos cansados del hombre." (Nietzsche,2005, p. 58).

En el Segundo Tratado de La Genealogía de la Moral, se trata de desentrañar el origen y la procedencia de la mala conciencia y la culpa. Nietzsche se sirve nuevamente del origen prehistórico de la relación contractual entre acreedor y deudor. En este mecanismo se origina la necesidad de crearle al deudor una memoria. El olvido, que fue ya anunciado por Nietzsche en De la utilidad y los inconvenientes de la Historia para la vida como una fuerza activa, sana y fecunda se complementa a su vez con la capacidad de recordar. Así, se entiende que es necesaria una memoria ¿Para qué? para poder realizar promesas, quien tiene una deuda debe poder recordar dicha falta a fin de cumplirla. A un posible incumplimiento, la sociedad impone al deudor toda la fuerza de su castigo. Asimismo, Nietzsche ve en este procedimiento el origen de toda idea acerca de lo divino. Los hombres sienten para con sus antepasados una deuda, propia de quien debe la subsistencia de su estirpe por los sacrificios realizados por sus antepasados. (Nietzsche, 2005, p. 144). El monoteísmo cristiano es el gran ejemplo de una deuda eterna con Dios Padre, la deuda y luego la falta y la culpa por el hecho mismo de existir, el pecado original. El segundo tratado, ofrece entonces, la psicología de la conciencia y de la culpa, ésta que fue siempre el instinto de la crueldad que cuando al tornarse incapaz de exteriorizarse se introyecta. Nos brinda Nietzsche su explicación de una idea de lo divino como deuda con sus antepasados. Ésta, en sus variados matices, se disfraza y toma la forma de la voz de Dios en el hombre, este proceso es algo inseparable del desarrollo cultural del hombre.

El tercer tratado, se encarga específicamente del problema del ideal ascético. El autor también tiene una mirada ambigua sobre el mismo; por ejemplo, este variará en tanto se sirvan de él filósofos, artistas, sacerdotes, etc. En primer lugar se puede decir 
que el ideal ascético forma parte de un instinto de protección y conservación. El problema aparece cuando éste adopta la forma de una vida que degenera y debilita la potencia vital. En un comienzo, menciona Nietzsche, el ideal ascético le sirvió a los primeros filósofos para dar sus primeros pasos en el camino de la filosofía. En un ambiente en que los ideales reinantes eran otros, el sabio antiguo producía cierta preocupación, manifestación de una vita contemplativa orientada a la reflexión necesitó de un nuevo ideal a partir del cual hacerse respetar y temer. Adoptó entonces la crueldad para consigo mismo y la ascesis como renuncia y sacrificio se instauró. (Nietzsche, 2005). En un sentido más propio, el ideal ascético sirve al filósofo porque ve en él las condiciones óptimas para el ejercicio de la filosofía, un puente hacia la independencia.

Este ideal es de suma preocupación para Nietzsche puesto que es aquel que triunfó en la historia, el único hasta el momento. Para el filósofo:

(...) el ideal ascético nace del instinto de protección y de salud de una vida que degenera, la cual procura conservarse con todos los medios, y lucha por conservarse; es indicio de una paralización y extenuación fisiológica parciales, contra las cuales combaten constantemente, con nuevos medios e invenciones, los instintos más profundos de la vida, que permanecen intactos. (Nietzsche, 2005, p. 155)

Esto se trasluce en todas las metafísicas, las religiones, las morales de la renuncia y en especial en la figura del sacerdote asceta que ve en ellas el dispositivo clave para ejecer su dominio. El sacerdote, dotado de una intuición especial para observar la inseguridad y sufrimientos propios de la vida de los individuos, les ofrece un consuelo pero brinda la solución que les brinda es más perjudicial que la enfermedad misma. Un veneno contra la vida: 
Abril 2020.

Tiene que ser una necesidad de primer rango la que una y otra vez hace crecer y prosperar esta especie hostil a la vida, - tiene que ser, sin duda, un interés de la vida misma el que tal tipo de autocontradicción no se extinga. Pues una vida ascética es una autocontradicción: en ella domina un resentimiento sin igual, el resentimiento de un insaciado instinto y voluntad de poder que quisiera enseñorearse, no de algo existente en la vida, sino de la vida misma, de sus más hondas, fuertes, radicales condiciones; en ella se hace un intento de emplear la fuerza para cegar las fuentes de la fuerza; en ella la mirada se vuelve, rencorosa y pérfida, contra el mismo florecimiento fisiológico, y en especial contra la expresión de éste, contra la belleza, la alegría (Nietzsche, 2005, p. 152)

La forma de valoración del asceta deviene de esta manera de interpretar el mundo. Hay una valoración negativa de la vida, en la que esta vida solo es admitida en tanto negación o como puente hacia otro mundo. Valoración del resentimiento y de la renuncia, que va en contra de los sentidos. Busca y encuentra el error en aquello que es más natural y siente como real: el cuerpo, los sentidos, los instintos, etc. Nace del instinto de protección y de salud de una vida que degenera, aún así "esa voluntad necesita una meta - y prefiere querer la nada- a no querer. " (Nietzsche, 2005, p.154)

\section{El concepto de vida nietzscheano, clave en el debate biopolítico actual}

Ante el incesante triunfo del ideal ascético dirá que si bien este es el ideal por excelencia de un estado de decadencia cultural, este ha triunfado y debe gran parte de su poder, a que "ha sido hasta ahora el único ideal no ha tenido ningún competidor" (Nietzsche, 2006, p.122). Lejos de una lectura pesimista y de renuncia ante esto, que de lo contrario lo ubicaría en el mismo horizonte de aquello que intenta superar, Nietzsche va a esbozar a lo largo de su obra una nueva filosofía que él llamará una filosofía del futuro. Para eso es necesario, un contraideal: 
Ese hombre del futuro, que nos liberará del ideal existente hasta ahora y asimismo de lo que tuvo que nacer de él, de la gran náusea, de la voluntad de la nada, del nihilismo, ese toque de campana del mediodía y de la gran decisión, que de nuevo libera la voluntad, que devuelve a la tierra su meta y al hombre su esperanza, ese anticristo y antinihilista, ese vencedor de Dios y de la nada - alguna vez tiene que llegar... (Nietzsche, 2005, pp. 123-124)

Podemos decir que el ideal del superhombre, deviene necesariamente de la idea expuesta extensamente en el aforismo 125 de La ciencia jovial, en el cual se formula la muerte de Dios, a esta idea la podemos interpretar de manera simple como toda pérdida de fe y creencia en los viejos ideales de una moral decadente, reflejado en el orden social, jurídico, político, científico, etc. El superhombre, en voz de Zaratustra viene a proponer esa unificación que estaba escindida en el hombre, es por eso que declaramos aquí que la muerte de Dios necesariamente significa la muerte el hombre tal y como era concebido hasta el momento.

¡Mirad, yo os enseño el superhombre! El superhombre es el sentido de la tierra. Que vuestra voluntad diga: iel superhombre es el sentido de la tierra! Yo os conjuro, hermanos míos, permaneced fieles a la tierra y no creáis en quienes nos hablan de esperanzas ultramundanas! (...) Son despreciadores de la vida moribundos, envenenados por su propia mano, de quienes la Tierra está cansada: iojalá desaparezcan! (Nietzsche, 2014, p. 21)

Este contraideal, marca la ruptura con toda metafísica en la historia de la filosofía y en especial con todo ideal cristiano. La tierra como eje de gravitación, es el punto sobre el cual mediante una perspectiva inmanentista se deberán crear todos los nuevos sentidos y valores. Lejos de buscar el sentido en un mundo ideal carente de realidad, Nietzsche ubica el sentido terrenal como suficiente en sí mismo. Esta nueva manera de 
Abril 2020.

ISSN 2344-9934

transvaloración, rompe con una forma de conocer que despreciaba los sentidos y la corporalidad como fuentes ricas de conocimiento, ante las antiguas concepciones gnoseológicas Nietzsche (2005) propone el perspectivismo:

Existe únicamente un ver perspectivista, únicamente un «conocer» perspectivista; y cuanto mayor sea el número de afectos a los que permitamos decir su palabra sobre una cosa, cuanto mayor sea el número de ojos, de ojos distintos que sepamos emplear para ver una misma cosa, tanto más completo será nuestro "concepto» de ella, tanto más completa será nuestra «objetividad». Pero eliminar en absoluto la voluntad, dejar en suspenso la totalidad de los afectos, suponiendo que pudiéramos hacerlo: ¿cómo?, ¿es que no significaría eso castrar el intelecto? (p. 155)

Lo expresado en este ideal que brota de una fuerza vital fecunda y ascendente es "la afirmación del fluir y del aniquilar, que es lo decisivo en la filosofía dionisíaca, el decir sí a la antítesis y a la guerra, el devenir, el rechazo radical incluso del concepto mismos de <ser>" (Nietzsche, 2006, p. 79). En este sentido, corresponde la preguntarse qué rol le cabe al ideal ascético en todo esto. Es ya conocido y lo hemos dicho, que el ideal ascético adopta diferentes formas. En el tercer tratado de la genealogía Nietzsche es muy claro en distinguir un ideal ascético propio de la décadence del ideal del cual se sirvieron por ejemplo los antiguos sabios o los mismos artistas. El punto a resaltar es que este ideal parte de un instinto de conservación propios de la vida y la voluntad de poder. En este sentido esta necesidad de autodefensa y autoconservación no debe leerle solo en términos negativos, ella existe pero no puede construir la finalidad propia del individuo, sino que debe estar orientada a otra cosa. Su exceso es sin dudas un peligro para la vida misma, en otras palabras: "Simplemente por la continua necesidad de defenderse puede uno llegar a volverse tan débil que ya no pueda defenderse." (Nietzsche, 2006, p.56).

A este punto se hace necesario entonces expresar qué significa la vida para nuestro autor. En uno de sus escritos tempranos, Nietzsche (2002) define la vida como “(...) esa potencia oscura, impulsiva, insaciablemente ávida de sí misma. Su veredicto 
es siempre inclemente, siempre injusto (...)" (р. 38). Otros estudiosos como Cano, llegan a identificar la noción de vida nietzscheana con lo que el autor denomina en una de sus Intempestivas con el nombre de fuerza plástica. Nietzsche (2002) refiere a la misma como “(...) esa fuerza para crecer desde la propia esencia, transformar y asimilar lo que es pasado y extraño, cicatrizar las heridas, reparar las pérdidas, rehacer las formas destruidas." (p .22). Pero como es sabido, este estilo metafórico no siempre es el más adecuado a la hora de buscar definiciones claras y precisas. Las nociones de autoconservación y superación tienen aquí gran importancia y se hace necesario un análisis más complejo.

Consideraremos entonces la lectura hecha de un texto Marina García Granero titulado Nietzsche y el mejoramiento humano.... En dicho escrito la autora se encarga de plasmar específicamente cómo podría interpretarse en claves nietzscheanas un mejoramiento de la especie humana a partir de la noción de vida que el autor brinda. En este sentido polemiza constantemente con algunas ideas de Charles Darwin, científico leído por Nietzsche y que este último tiene en cuenta en sus formulaciones aunque la mayoría de las veces sin coincidir con él. Siguiendo con esto, el principal concepto que cuestionará Nietzsche al científico será el de selección natural, para el alemán la selección natural:

no ejerce un fenómeno de selección, sino únicamente un movimiento de adaptación: no se realiza ningún refinamiento cualitativo, sino que a través del proceso adaptativo de los organismos respecto al medio que habitan, se tiende a la eliminación de caracteres excepcionales. (García Granero, 2017, p. 602)

Dicho esto, es necesario entender que más allá de las consecuencias científicas y la validez de la crítica nietzscheana a dicha teoría, lo que Nietzsche intenta denunciar son los supuestos culturales e ideológicos bajo los que se sustenta dicha formulación. Nietzsche no ve en la conservación y adaptación de la especies los fines más altos a los 
Abril 2020.

que pueda llegar una cultura. La selección natural supone para Nietzsche la adaptación de un gran número de individuos con caracteres similares que imposibilitan la supervivencia y desarrollo de aquellos caracteres más singulares y elevados. En esta línea, sigue presente la pregunta sobre la utilidad y la finalidad de los conceptos y los valores ya puesta en cuestión en La Genealogía de la Moral. En palabras de Nietzsche, en Más allá del Bien y del mal expresa "El ser vivo quiere ante todo dar libre rienda a su fuerza. La vida misma es voluntad de poder. El instinto de conservación no es más que una consecuencia indirecta, una de las más frecuentes." (Nietzsche, 2014, p. 398).

Es posible pensar entonces en otro tipo de finalidad de la cultura que sea superior al de la mera supervivencia y conservación. Si se lee a Nietzsche a partir de estos elementos no sorprende entonces, sus tantos aforismos orientados a la misma cuestión, la ya conocida temática de la cultura y la producción del genio por ejemplo. Nietzsche ve en los griegos por ejemplo, el caso por antonomasia de la manifestación del genio cultural. El punto más polémico en esto, es la defensa de una tesis que sostiene que la realización de la excelencia cultural sólo es posible bajo condiciones desiguales de existencia. Como ilustración a este problema, citamos in extenso el aforismo 235 de Humano, demasiado Humano:

Genio y Estado ideal en contradicción: Los socialistas desean establecer el bienestar para el mayor número posible. Si la patria perdurable de este bienestar, es Estado perfecto, fuese realmente alcanzada, el bienestar destruiría el terreno donde la gran inteligencia y, generalmente, la individualidad poderosa: me refiero a la energía recia. La humanidad sería demasiado inerte, una vez realizado este Estado, para producir aún el genio ¿No habría entonces que desear, por esta razón, que la vida conserve su carácter violento y que incesantemente sean llamadas a la vida de nuevo fuerzas y energías salvajes? (Nietzsche, 1984, p.177) 
Este último punto, en conjunto con el problema del ideal ascético, lo retomaremos en el siguiente capítulo para establecer algunos aportes y discusiones que se pueden entablar entre la biopolítica y su relación con la tanatopolítica entre Nietzsche y Espósito.

Aportes de Nietzsche al debate entre biopolítica y tanatopolítica. ¿Es posible hablar de una biopolítica afirmativa y negativa en Nietzsche?

Nietzsche ve en la cultura occidental la constante imposición del ideal ascético, su incesante triunfo en todos los ámbitos. Diremos que Nietzsche se ubica en muchos aspectos como un crítico de la cultura y muchas veces en rol de un filósofo-médico. El diagnóstico es harto conocido: la sociedad occidental, especialmente identificada con la Europa moderna está enferma. Solo ha reinado hasta ahora es espíritu de la décadence y el resentimiento. Nuestro pequeño aporte al campo de la biopolítica tiene que ver en este caso, con señalar que la sociedad que en Espósito se describe sigue perteneciendo a este período nihilista. El filósofo alemán ve en los Estados modernos un intento no solo de conservación de la población sino en eso mismo su decadencia, el intento de igualar sea ante el Estado, las leyes o las normas a los individuos no puede ser más que una política negativa para la cultura desde la visión de Nietzsche.

Si hablamos en términos de Esposito diremos lo siguiente: si en el campo de la biopolítica se trata de pensar la vida como aspecto a conservar y se ve a la tanatopolítica como dimensión que es pensada en términos negativos, esto no sucede en Nietzsche. Para Nietzsche, la vida encuentra su mayor enemigo en la decadencia, no en la muerte o en el peligro. La violencia, la guerra, las muertes no son en las teorizaciones nieztcehanas aspetos esencialmente negativos, estos potencias la vida, pues hacen aparecer a los caracteres más fuertes. Si en la biopolítica lo que se quiere evitar es su revés tanatopolítico, en Nietzsche lo que se intenta evitar es una excesiva autoconservación que vuelva inerte la vida misma en su capacidad cultural.

Es interesante ver, cómo en los autores biopolíticos existe esta preocupación incesante en determinar qué es lo que genera el pasaje de una política de la vida a una 
Abril 2020.

sobre la vida. En un Estado que por ejemplo está orientado a la conservación y el bienestar de la sociedad, Nietzsche no ve un aspecto positivo sino un peligro. Pensemos por ejemplo en la cuestión del Estado y el trabajo, en una sociedad que se caracteriza por este aspecto y en donde la biopolítica ve en el trabajo un instrumento de desarrollo poblacional. Nietzsche nos dirá por ejemplo que filósofo, que el gran mérito del sacerdote asceta no es curar la enfermedad sino generarla y brindar pequeñas dosis de adormecimiento, de alivio del dolor, nunca la cura. El sacerdote alivia temporalmente el dolor que él mismo se encarga de autodireccionar en los individuos, la cultura del trabajo no escapa a esta lógica: "Está fuera de toda duda que una existencia sufriente queda así aliviada en un grado considerable: a este hecho se le llama hoy, un poco sinceramente, "la bendición del trabajo»." (Nietzsche, 2005, p. 173). Y agrega:

La actividad maquinal y lo que con ella se relaciona -como la regularidad absoluta, la obediencia puntual e irreflexiva, la adquisición de un modo de vida de una vez para siempre, el tener colmado el tiempo, una cierta autorización, más aún, una crianza para la «impersonalidad», para olvidarse a-sí-mismo, para la incuria sui [descuido de sí]-: ide qué modo tan profundo y delicado ha sabido el sacerdote ascético utilizar estas cosas en la lucha contra el dolor! (Nietzsche, 2005, p.173)

Retomemos entonces, consideramos aquí un aporte clave para el campo biopolítico los estudios realizados en la Genealogía de la Moral de Friedrich Nietzsche, particularmente porqu realiza una interpretación completamente novedosa de los valores imperantes hasta la época de los cuales devienen muchos de los órdenes sociales y políticos de los Estados modernos. Sobre este tema el autor dirá: "la moral misma entendida como síntoma de décadence es una innovación, una singularidad de primer rango en la historia del conocimiento". (Nietzsche, 2006. p. 77). Un ejemplo de esto es el rasgo de corte socialista de algunos de ellos, que en pos de la igualdad y bienestar representan un peligro efectivo para la realización de individuos singulares y 
excepcionales. Ante esto Nietzsche no duda en anteponer para la excelencia cultural la necesidad de la violencia como dimensión constitutivamente vital y propicia para el desarrollo de la cultura. Coincidiremos entonces con Cano (2014) cuando señala que:

"Profundizando en esta crítica del "tú debes", Nietzsche trata de acceder precisamente a una consideración inédita, extra moral, de la verdad y del conocimiento: admitir que la no verdad es condición de la vida significa, desde luego enfrentarse de modo peligroso a los sentimientos de valor habituales." (p. 53)

Por lo mismo, consideramos que, tanto la obra como el método genealógico que inaugura y la nueva visión perspectivista del conocimiento como aportes indispensables a la hora de pensar y estudiar la vida y su relación con el poder. Varias razones se pueden lanzar para sostener la importancia de estos aportes, por un lado porque Nietzsche hace de la vida su objeto de estudio pero así también su propio sujeto, en el perspectivismo nietzscheanos es la voluntad de poder misma quien interpreta, son los sentidos, el cuerpo como una gran razón, los instintos quienes producen el conocimiento. En este sentido, la vida en todas sus dimensiones conscientes e inconscientes, decadentes y ascendentes, en su voluntad de mando y obediencia es la que se encuentra en el centro de la cuestión. Tal vez el mayor aporte del autor sea esta interpretación y esta perspectiva tan cabal y radical sobre la vida y los valores, la descripción de la vida en muchas de sus dimensiones es un aporte ineludible de la obra nietzscheana al campo biopolítico.

Esto último, nos da el pie para realizar unas breves relaciones sobre la cuestión tan discutida y paradojal entre biopolítica y tanatopolítica. Si entre los autores que retomamos en esta temática, es decir Esposito y su lectura de Foucault resulta un problema de difícil solución es la relación entre biopolítica y tanatopolítica, en Nietzsche encontramos algunas coordenadas que nos permiten algunas reflexiones sobre la misma. Es importante recordar que para Nietzsche la vida es inseparable de la 
Abril 2020.

violencia y la injusticia, así por ejemplo, ve en la guerra -que en términos políticos sería enmarcado en el horizonte de la tanatopolítica- un elemento indispensable de la cultura. En el aforismo 439 de Humano, demasiado humano el autor hará una apología de la guerra como el único medio capaz de devolver a los pueblos fatigados y en decadencia, la ruda energía de la batalla. Así por ejemplo citando el caso de la sociedad inglesa que parece empeñada en sustituir la violencia de la guerra mediante otras formas de aventura, dirá:

“(...) se inventarán en formas diversas sustitutivos parecidos de la guerra pero tal vez nos harán cada vez más ver una humanidad así educada, y por eso mismo tan fatigada como lo está hoy mismo Europa, tiene necesidad no solo de guerras, sino de las guerras más terribles - $y$, por tanto, de retornos momentáneos a la barbarie- para no gastar en medios de civilización su civilización y existencia mismas." (Nietzsche, 1984, p.268)

Independientemente de los análisis políticos y sociológicos que se puedan realizar y sin ánimos de tomar este vaticinio de Nietzsche como un destino inevitable de la Europa del siglo XX, lo que no se le puede negar al autor es su acierto en esta cuestión. Nietzsche vio en esa sociedad tan culta y lugar de la biopolítica, un elemento de potencial peligro, un exceso de conservación y adormecimiento, de fatiga fisiológica y cultural que el único remedio para evitar la disolución misma de ella era la vuelta a un estado de violencia. Cabe aclarar, como ya hicimos con otros conceptos que Nietzsche no ve en el Estado un mal en sí mismo para las individuos más sobresalientes. Sin embargo, cuando el rol del Estado es exagerado hasta su ennoblecimiento, vuelve a los individuos y la población toda inertes y débiles para la las grandes acciones y la cultura superior. (Nietzsche, p.1984).

Como último punto de este capítulo y para finalizar la exposición, cerraremos con el objetivo restante de este trabajo la vinculación de Nietzsche con una biopolítica afirmativa o negativa. Si tenemos en cuenta todo lo dicho hasta ahora, debemos decir 
que Nietzsche es un autor que brinda muchos elementos que son fructíferos para los estudios biopolíticos. Por otro lado, en los términos en que trabajamos la cuestión de la biopolítica en Esposito, la obra nietzscheana adquiere a nuestro modo de entender un rasgo necesariamente negativo. Para esta argumentación nos servimos de nuestro capítulo denominado El concepto de vida nietzscheano, clave en el debate biopolítico actual y diremos que según nuestra lectura, es necesario distinguir los términos políticos en lo que actualmente se entabla el debate sobre la biopolítica y una ética nietzscheana que sí consideramos afirmativa. En una de sus obras más importantes: Comunitas origen y destino de la comunidad, Espósito al hablar de Hobbes menciona la necesidad de distinguir el campo de la ética y de la política, decimos lo mismo de Nietzsche. En términos políticos nuestro autor reviste un carácter negativo, al menos en las claves en las que se enmarca el debate actual sobre la biopolítica, así como en sus objetivos de la protección y la conservación de la vida de la población. En cuanto al aspecto ético, la filosofía nietzscheana es un abierto decir sí a valores que autor considera elevados y potencial la vida y en sentido terrenal, como pudimos ver a partir de Zaratustra y el superhombre, pero esta siempre está orientada a individuos excepcionales, no existe un intento de igualación entre individuos y por lo mismo carece de fuerza si se lo piensa en términos poblacionales o de masa.

\section{Conclusiones}

Para cerrar, luego del recorrido hecho hasta aquí mencionaremos brevemente los aportes que la lectura de parte de la obra de Nietzsche nos brindó al problema de la biopolítica y tanatopolítica.

En primer lugar, Nietzsche es un autor que se posiciona como crítico cultural, en este sentido la interpretación de la sociedad moderna y contemporánea hasta siglo XX como decadente pone en cuestión muchos de los conceptos modernos imperantes hasta la época. Podemos mencionar brevemente la propia moral decadente que genera un nihilismo cultural a partir del cual Nietzsche intenta dar una nueva 
Abril 2020.

alternativa. Este aspecto es importante porque pone en jaque las concepciones modernas que devienen de la moral, tales como el Estado, la religión, la política, el sistema jurídico, etc.

Por otro lado, la obra de Nietzsche brinda una interpretación radical y sumamente elaborada acerca de la sociedad de su tiempo y lo hace poniendo en el centro de gravitación la noción de vida misma. Propone una nueva filosofía a partir de un ideal o mejor dicho, de un contraideal que venga a competir con el único ideal reinante hasta el momento que es el ideal ascético. Así la filosofía dionisíaca pone en cuestión el concepto mismo del ser y declara la necesidad de una nueva forma de vida y una ciencia que responda a autosuperación y vinculación con la misma. Recogemos entonces la Genealogía de la Moral como una gran suma de contenido en cuanto a aportes teóricos para pensar una crítica cultural en el campo biopolítico y a su vez una interpretación de la noción de vida. Reconocemos la importancia que tiene en esto la genealogía como método de interpretación y valoración de ideales en unión con el aspecto fisiológico y en comunión con el mejoramiento cultural, así como también la importancia del perspectivismo como forma de conocimiento.

En cuanto a la relación entre biopolítica y tanatopolítica, hemos mencionado que la preocupación de Nietzsche radica más en el peligro del nihilismo y la decadencia cultural que en la cuestión de la muerte o el peligro. Reflexionamos largamente en el último capítulo acerca de estas concepciones nietzscheanas en torno a conceptos como el trabajo y la guerra. En este sentido, nos parece que Nietzsche brinda una perspectiva muy particular del tema que nos permite pensar esta relación por fuera de un revestimiento negativo de la tanatopolítica, pues el autor ve en varios fenómenos de la misma, condiciones indispensables la cultura. Terminamos con la afirmación de que si bien no podemos hablar de Nietzsche como biopolítico afirmativo, este propone muy coherentemente con sus pensamientos una ética que sí resulta afirmativa. Por lo mismo, esta necesidad de distinción entre ética y política también nos resulta un aporte importante para el campo. 
Concluimos diciendo que una lectura de Nietzsche orientada a aportar a la relación entre biopolítica y tanatopolítica es sumamente rica pues permite una mirada desde afuera, que excede a la discusión entre una tendencia biopolítica negativa o afirmativa de la misma. Esta mirada desde afuera, permite ver, que desde la crítica del ideal ascético llevada a cabo por Nietzsche se puede criticar a su vez el ideal de una biopolítica que funda todos sus esfuerzos en el objetivo de la conservación, legislación y regulación de la vida poblacional. No se trata de denunciar que las preocupaciones de Esposito por el devenir de una biopolítica en tanatopolítica no sean relevantes, ni tampoco se trata de insistir en que la preocupación por la conservación de la seguridad de la población no revista un gran interés, sino en que visto este panorama desde la mirada nietzscheana, este no puede ser el único objetivo de la política y de la ética.

\section{Referencias bibliográficas}

- Cano, G. (2014). Estudio introductorio. En: Editorial Gredos (Ed.), Nietzsche I. (pp. 7-101). Barcelona: Editorial Gredos.

- Cragnolini, M. (2011). La cuestión de lo posible y la potencia en la biopolítica. En: Instantes y Azares, Año XI, Nro. 9, pp.151-156.

- Cragnolini, M. (2012). Apuntes para un pensar que no se agota: Nietzsche y biopolítica. En: E. Díaz (Ed.), El poder y la vida: modulaciones epistemológicas. (pp. 19-31). Buenos Aires: Biblos.

- Esposito, R. (2003). Comunitas: origen y destino de la comunidad. Buenos Aires: Amorrortu.

- Espósito, R. (2006). Bíos. Biopolítica y Filosofía. Buenos aires: Amorrortu.

- Foucault, M. (2007). La Historia de la Sexualidad I. La voluntad de saber. México: Siglo XXI Editores.

- García Granero, M. (2017). Nietzsche y el mejoramiento humano. Reflexiones en torno a la noción de vida. ISEGORíA. Revista de Filosofía Moral y Política. № 5, pp. 599-615. doi: 10.3989/isegoria.2017.057.09

- Nietzsche, F. (1984). Humano, demasiado humano. Madrid: EDAF. 
- Nietzsche, F. (2002). Consideraciones Intempestivas. Buenos Aires: Alianza. Editorial.

- Nietzsche, F. (2005). La Genealogía de la Moral. Buenos Aires: Alianza Editorial.

- Nietzsche, F. (2006). Ecce Homo. Cómo se llega a ser lo que se es. Buenos Aires: Alianza Editorial.

- Nietzsche, F. (2014). La ciencia jovial. Barcelona: Editorial Gredos.

- Nietzsche, F. (2014). Así habló Zaratustra. Barcelona: Editorial Gredos.

- Nietzsche, F. (2014). Más allá del bien y del mal. Barcelona: Editorial Gredos.

- Vergara Henríquez, F.J. (2001). El "sacerdote asceta" y el sacro-dominio del valor. Nietzsche y la genealogía de la moral. Estudios de Filosofía, no , 43, pp. 129-148. Recuperado de http://www.scielo.org.co/pdf/ef/n43/n43a07.pdf 